\title{
User Effects on MIMO Performance: From an Antenna to a Link Perspective
}

\author{
Shirook M. Ali, Amin Mobasher, and Paul Lusina \\ Advanced Technology, Research In Motion Limited, Water loo, ON, Canada N2L 3W8 \\ Correspondence should be addressed to Shirook M. Ali, shali@rim.com
}

Received 18 January 2011; Revised 9 May 2011; Accepted 15 June 2011

Academic Editor: Zhongxiang Q. Shen

Copyright () 2011 Shirook M. Ali et al. This is an open access article distributed under the Creative Commons Attribution License, which permits unrestricted use, distribution, and reproduction in any medium, provided the original work is properly cited.

\begin{abstract}
We investigate in this paper the effects of the user's presence on the performance of a multiple-input multiple-output (MIMO) system in data and in voice usage scenarios. The investigation studies the user effects on the antenna performance and how these are incorporated into the MIMO channel and the link characteristics. The antennas and the user are deterministic. These are then integrated into the statistical 3GPP spatial channel model (SCM) for a typical macrocell propagation environment setting. The channel performance is analyzed based on the average channel capacity, the average power transfer, the correlation, and the cumulative distribution function of the channel capacity as well as the link throughout and the error performance. The mentioned channel and link properties are tied to the MIMO antenna properties that are represented in the mutual coupling between the antennas, the power loss, the total radiated power, the mean effective gain (MEG), as well as the efficiency with emphasis on how the user affects each. It was found that the presence of the user contributed to a loss of up to $50 \%$ in the average channel power transfer. The data position was found to be the lowest in terms of channel capacity performance. The voice position performance showed a large dependence on the user orientation with respect to the line of sight path while the data position showed less dependence on the user's orientation. We also discuss through the examined antenna and channel properties the importance of the channel multipath on the MIMO performance. In some scenarios, it was found that a well-conditioned channel can compensate for losses due to the presence of the user, improving the overall system performance. The presented investigation at the link level also discusses the user effects in different MIMO transmission schemes.
\end{abstract}

\section{Introduction}

There is growing research activity investigating the interaction between the electromagnetic (EM) fields radiated from a wireless device and the human tissues. These studies generally deal with two aspects. The first aspect is the absorption of the microwave energy by the user's body, which is significant from a safety standards point of view. The second aspect is the influence of the user's body on the radiation properties of the mobile phone, which is significant from the antenna design point of view. The investigation presented in this paper focuses on the second aspect, the user effects on the wireless antenna device performance.

Despite the potential of multiple-input multiple-output (MIMO) systems [1], recent studies have shown that there are severe limits to the theoretical ergodic MIMO capacities when more realistic scenarios are accounted for [2]. Factors influencing performance in real MIMO fading scenarios include a realistic propagation channel, mutual coupling between the antennas, the antenna radiation efficiencies, and the presence of the user, among other factors. While the EM field-human body interaction has been thoroughly investigated in the literature, the effects of the presence of the user on an MIMO performance have not received much attention. The presence of the user is shown to have an immediate influence on the radiation patterns, input impedances, and therefore, on the correlation matrix and the overall system performance. Yet, the effects are not fully investigated.

The effect of the user on the performance of a single antenna system has been thoroughly discussed in [3-5]. These indicated that significant gain reduction occurs when the antenna is used in the user's vicinity, and approximately half of the transmission power is absorbed in the human 


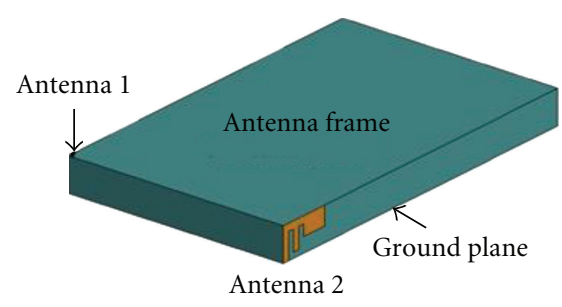

(a)

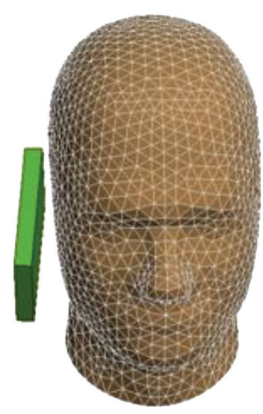

(b)

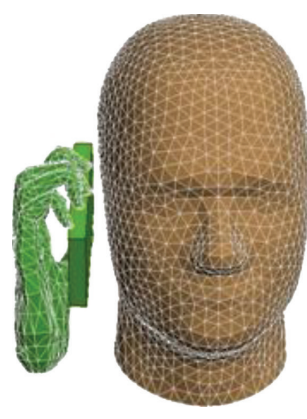

(c)

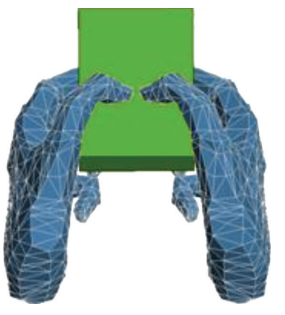

(d)

FIGURE 1: Simulation models for usage scenarios: (a) free space, that is, no user, (b) head and handset in tilt position, (c) voice position (head, hand, and handset in tilt position), (d) data position (two hands and handset).

TABle 1: Tissue properties and their assumed percentage in the hand model.

\begin{tabular}{lccc}
\hline Tissue type & Permittivity & $\begin{array}{c}\text { Conductivity } \\
(\mathrm{S} / \mathrm{m})\end{array}$ & $\begin{array}{c}\text { Assumed } \\
\text { percentage in the } \\
\text { hand model (\%) }\end{array}$ \\
\hline Muscle & 53.3 & 1.9 & 40 \\
Bone & 14.8 & 0.6 & 40 \\
Skin (dry) & 37.8 & 1.5 & 20 \\
\hline
\end{tabular}

body, resulting in degradation in the radiation efficiency. The influence due to the hand occurs not only in the talk mode, but is also significant in the data mode (hands in front of body). In [5], the influence on the antenna performance criteria of the position and the grip of the hand on the phone in data mode was investigated to support the standardization process of the hand phantom, and to propose a method to analyze the influence due to the hand.

The interest in studying the user effects on the performance of a multiantenna system is very recent; the performance of these systems is usually reported without the user. Generally, the effects of the user have been investigated on the performance of the antenna and have not been connected to the change on the link performance. A reduction in the mean effective gain (MEG) due to the presence of the user has been reported $[6,7]$. The user can have a different effect on each of the MIMO antennas in the mobile device depending on the usage scenario. This causes a gain imbalance that could also affect the overall system performance.

In this work we examine the MIMO performance as applied to the user effects from an antenna perspective and incorporate these into a channel model for link analysis. A MIMO two-antenna design based on a planar inverted-F antenna (PIFA) is considered. For this, the antenna radiation properties as defined in the presence of the user and in the different usage scenarios is included in the spatial channel model (SCM) [8], which is a statistical channel model. The performance is studied through the channel statistics, namely, the average channel capacity, the outage capacity, the correlation, the channel power factor, and the cumulative distribution function of the channel capacity. The investigation is extended to investigate the user effects at a link level. The investigated antenna parameters include impedance match, mutual coupling, power loss, efficiency, the MEG, and radiation pattern for each antenna in the handset. Two usage scenarios are considered: (1) voice positions - tilt position recommended by IEEE Std. 1528 [9], and (2) data position when two hands are considered in the vicinity of the handset. Phantom head and hands are used with materials set from [10]. It is worth mentioning here that the paper does not aim at providing design guidelines. The focus is on providing comprehensive analysis for a typical two-antenna handset device.

The remainder of the paper is organized as follows. The problem set-up describing the deterministic and the statistical parts of the integrated channel model are introduced in Section 2. Section 3 presents the results and the discussion. The user effects on the antenna properties and the capacity statistics of the channel are also introduced in Sections 3.1 and 3.2, respectively. The link analyses are presented in Section 3.3. Conclusions are given in Section 4.

\section{Problem Definition}

To incorporate the effects of the user and the antennas, we assume that the antennas and the user's near-field are incorporated in the channel matrix, $\mathbf{H}$. The channel matrix relates the complex input vector $\mathbf{x}$ to the complex output vector $\mathbf{y}$ during a symbol period after adding the white Gaussian noise vector $\psi$,

$$
\mathbf{y}=\mathbf{H x}+\psi
$$

The transmitted signal vector $\mathbf{x}$ is assumed to be measured at the antenna terminals and to have $N$ independent equally powered signals with no channel state information (CSI) at the transmitter. We consider the normalized channel matrix $\overline{\mathbf{H}}$ in (1) which is defined using the Frobenius normalization [11],

$$
\overline{\mathbf{H}}=\frac{\sqrt{N M}}{\|\mathbf{H}\|_{F}} \cdot \mathbf{H}, \quad \text { where }\|\cdot\| \text { is Frobenius norm }
$$

In our simulations, the channel matrix $\mathbf{H}$ is computed statistically for a typical macrocell environment but with 
TABLE 2: Channel models simulation parameters.

\begin{tabular}{lc}
\hline Parameter & Setting \\
\hline Carrier frequency & $2 \mathrm{GHz}$ \\
MS height & $1.5 \mathrm{~m}$ \\
BS height & $32 \mathrm{~m}$ \\
Channel state information & No CSI at the Tx and perfect \\
(CSI) & CSI at the Rx \\
BS to MS distance & $100 \mathrm{~m}$ \\
Number of realizations & 10,000 \\
Antennas' characteristics & Equivalent 2D of the embedded \\
Scenario & patterns, effective gain \\
User scenarios (Figure 1) & Typical macrocell \\
\end{tabular}

TABle 3: Percentage reduction (\%) in the efficiency with the different usage scenarios compared to the nonuser scenario.

\begin{tabular}{lcc}
\hline & Antenna 1 & Antenna 2 \\
\hline Head & 22 & 22 \\
Head and hand & 66 & 75 \\
Data position & 51 & 46 \\
\hline
\end{tabular}

TABLE 4: The MEG in DB evaluated for the MIMO design.

\begin{tabular}{lccc}
\hline & Antenna 1 & Antenna 2 & Average \\
\hline Without user & -3.4 & -3.5 & -3.4 \\
Head & -4.2 & -6.6 & -5.2 \\
Head and Hand & -4.6 & -9.6 & -6.4 \\
Data position & -6.4 & -6.4 & -6.4 \\
\hline
\end{tabular}

specific antenna properties on the mobile station side, that is, the antenna properties are deterministic. In the following subsections we provide insight on each of these parts, the deterministic and the statistical, that are integrated to form the complete channel.

2.1. The Deterministic Electromagnetic Model. The EM simulations are performed with a full-wave 3D solver, FEKO [12]. Figure 1(a) shows the simulation model of the MIMO handset. The handset has the dimensions of a typical smartphone of $100 \times 60 \times 12 \mathrm{~mm}^{3}$. Two classic single-band antennas based on the PIFA are designed copolarized to each other. Both antennas are designed to resonate at $2.6 \mathrm{GHz}$. The separation between the antenna feeds is equal to the width of the handset, that is, $60 \mathrm{~mm}$.

The examined usage scenarios are shown in Figures 1(b)$1(\mathrm{~d})$ where the phantom head and the hand models are used to simulate the user. In order to assign material properties to the homogeneous phantom head model at the frequency of $2.6 \mathrm{GHz}$ we use $[9,10]$. It is worth noting that the properties have not been explicitly reported at this frequency. Therefore, we interpolate between the closest frequencies of $2.45 \mathrm{GHz}$ and $3 \mathrm{GHz}$ to obtain the material values at $2.6 \mathrm{GHz}$. This resulted in a permittivity $\mathcal{E}_{r}$ of 39 and a conductivity $\sigma$ of $1.96 \mathrm{~S} / \mathrm{m}$ in the measurements.

Furthermore, to assign properties to the homogeneous hand model, we assume that the hand is made of $40 \%$ bone, $40 \%$ muscle, and $20 \%$ skin. Then we use the properties provided in [9] by Federal Communications Commission (FCC). Table 1 shows the electromagnetic properties and the percent composition that each material has been assigned to give the average properties of the homogeneous hand model. The average properties of $\varepsilon_{r}=34.8, \sigma=1.3 \mathrm{~S} / \mathrm{m}$ are then assigned to the hand model. These seem to agree well with the physical head and hand phantoms used.

A single full-wave EM simulation of the antenna system is required to obtain the field patterns that describe the antenna's behaviour taking into account any near-field obstacles such as the user's head and or hand(s). These 3D patterns are obtained using the FEKO simulation software, and are then integrated into the SCM channel model as shown in Figure 2.

2.2. The Statistical Channel Model-SCM. In the SCM [8], the spatial and temporal distributions are parameterized to characterize the channel in the urban macrocell environment. One realization consists of six paths composed of twenty subpaths each. The parameters of all subpaths are calculated for each realization. A temporal channel matrix $\mathbf{H}_{n}, n=\{1 \cdots 6\}$, is created by the SCM program for each cluster through the superposition of the subpaths. The entries $h_{i, j, n}$ of $\mathbf{H}$ are

$$
\begin{aligned}
& h_{i, j, n_{p}} \\
& =\sqrt{\frac{P_{n_{p}} \delta_{\mathrm{SF}}}{20}} \\
& \quad \times\left(\sum_{m_{s}=1}^{M_{s}} \sqrt{G_{\mathrm{BS}}\left(\theta_{n_{p}, m_{s}, \mathrm{AoD}}\right)}\right. \\
& \quad \times \exp \left(J\left[k d_{j} \sin \left(\theta_{n_{p}, m_{s}, \mathrm{AoD}}\right)+\Phi_{n_{p}, m_{s}}\right]\right) \\
& \left.\quad \times \sqrt{G_{\mathrm{MS}_{i}}\left(\theta_{n_{p}, m_{s}, \mathrm{AoA}}\right)} \exp \left(J\left(k d_{i} \sin \left(\theta_{n_{p}, m_{s}, \mathrm{AoA}}\right)\right]\right)\right), \\
& \quad \text { where } j=\sqrt{-1} .
\end{aligned}
$$

The indices $i$ and $j$ correspond to the receive and the transmit antenna elements, respectively. The number of paths is indexed by $n_{p}=\{1 \cdots 6\}$ and the number of subpaths are $m_{s}=\{1 \cdots 20\}$. The bulk of the parameters depend on large-scale effects of the environment determined by the pathloss factor $P_{n p}$ and the shadow fading factor $\delta_{\mathrm{SF}}$. The effects of the bulk parameters are removed via the normalization approach. The $M_{s}$ subpaths associated with the scatter fading are defined by the gain pattern of the MS and $\mathrm{BS}, G_{\mathrm{BS} j}\left(\theta_{n, m, \mathrm{AoD}}\right)$, and $G_{\mathrm{MS} i}\left(\theta_{n p, m s, \mathrm{AoA}}\right)$, where $\theta$ is the angle of arrival (AoA) and angle of departure (AoD), respectively. The phase of each subpath depends on the 
TABLE 5: The total radiated power (TRP) and power loss $\left(P_{L}\right)$ for PIFA (in DB).

\begin{tabular}{lcccc}
\hline & \multicolumn{2}{c}{ Antenna 1} & \multicolumn{2}{c}{ Antenna 2 } \\
& TRP & $\mathrm{P}_{\mathrm{L}}$ & TRP & $\mathrm{P}_{\mathrm{L}}$ \\
\hline Without user & -19.51 & -39.24 & -19.51 & -39.17 \\
Head & -20.31 & -25.67 & -20.14 & -25.65 \\
Head and Hand & -26.07 & -22.18 & -24.05 & -19.91 \\
Data position & -23.46 & -23.05 & -23.47 & -23.05 \\
\hline
\end{tabular}

TABLE 6: LTE link simulation parameters.

\begin{tabular}{|c|c|}
\hline Parameter & Setting \\
\hline MIMO mode & $\begin{array}{c}\text { Spatial multiplexing/transmit } \\
\text { Diversity }\end{array}$ \\
\hline Antenna configuration & $2 \times 2$ or $4 \times 2$ \\
\hline Equalization & MMSE \\
\hline Channel estimation & Known \\
\hline $\begin{array}{l}\text { Adaptive modulation and } \\
\text { coding }\end{array}$ & Enabled \\
\hline Coding rate & $1 / 2$ \\
\hline HARQ & Enabled (with two retransmission) \\
\hline Correlation & Low \\
\hline
\end{tabular}

geometry of the BS and MS linear array and is determined by $k d_{j} \sin \left(\theta_{n p, m s, \mathrm{AoD}}\right)$ and $k d_{i} \sin \left(\theta_{n p, m s, \mathrm{AoA}}\right)$, respectively. Here, $d_{j}$ and $d_{i}$ are the element separation of a uniform linear array at the BS and MS, respectively, and $k=2 \pi / \lambda$. A random phase term $\Phi_{n p, m s}$ is added to each subpath phase. Table 2 shows the default SCM simulation parameters for the urban macrocell.

2.3. The Integrated SCM-Electromagnetic Model. Figure 2(a) shows the orientation of the MS with respect to the BS. The analysis presented later in the paper considers the different orientations of the used and the handset as defined by the angle $\theta_{\mathrm{MS}}$.

The flowchart shown in Figure 2(b) shows the integration between the deterministic antenna properties and the statistical channel model. Since SCM is a two-dimensional (2D) channel model while the antenna field properties are in a three-dimensional pattern form, an equivalent transformation from a $3 \mathrm{D}$ pattern to an equivalent $2 \mathrm{D}$ pattern is required and is described in $[13,14]$. The antenna properties such as the effects of coupling and antenna gain are all preserved during this transformation.

2.4. Measurements. The investigated MIMO design in the vicinity of the user is simulated and their performance is also confirmed with measurements. The $S$-parameters as well as the radiation patterns were the measured parameters. The measurements for the $S$-parameters for voice and for data scenarios were performed inside the anechoic chamber. Homogeneous head and hand phantoms are used in the measurements that have almost the same electrical properties as those used in the simulations given in Table 1. Figure 3 shows the measurement set-up for both the voice and the data scenarios.

\section{Results and Discussion}

The presence of the user on the examined MIMO system performance is investigated first based on the antenna properties. The antenna properties are then incorporated into the channel model for channel and link level analyses. We describe these in detail in the following two sections.

3.1. Antenna Properties. Three aspects of the antenna performance are investigated in this section, the reflection and the transmission coefficients (mutual coupling between the antennas), the antennas' efficiencies, as well as the far-field radiation properties and power.

3.1.1. Return Loss and Coupling. Figure 4 shows Sparameters for the MIMO design obtained from simulations and from measurements. The reflection coefficient is given by $S_{11}$ while the coupling coefficient is given by $S_{21}$. The presented plots are for the cases with and without the user. The simulation and the measurement results are very good, despite the difficulty of matching the measurement set-up to the simulation environment. Notice that the return loss in the presence of the user only shifts $2 \%-3 \%$ around the resonance frequency of $2.6 \mathrm{GHz}$. The greatest shifts appear in the data position. The mismatch in the antenna impedance in the presence of the user translates into power reflected back into the front-end of the device transceiver.

The mutual coupling $S_{21}$ curves between the two antennas are shown in Figures 4(e) and 4(f) for the simulated and the measured results, respectively. Notice that, except for the scenario of the head position, coupling between the two antennas in fact improves, that is, decreases in the presence of the user head and hands. At first this might sound antiintuitive as the user hands wrap around the antennas and are expected to lock the radiated power around the antennas which would increase the $S_{21}$. value. However, the opposite result can be explained by noting that the presence of the user reduces the total radiated power (TRP). Hence the leakage of energy from one antenna into the other is now less-this will also be showed later in the paper when the radiation properties are discussed. Therefore, this reduction in coupling as a result of the human interaction is not necessarily seen as a positive effect. The same logic can be used to explain the increased coupling in the case of the head, which came from improved TRP (see the pink curves in Figures 4(e) and 4(d)).

3.1.2. Efficiency. Figure 5 shows the simulated variations of the efficiencies of the MIMO design antennas in the investigated scenarios. In all usage scenarios, the user's presence degrades the efficiency and in a much pronounced way than was noticed in the $S$-parameters' results of Figure 4 due to the loss in the user's tissue. As expected, the highest efficiencies, about 55\%, among all cases is found to be without the user's presence. The percentage decrease of 
the efficiency values compared to the free space case is tabulated in Table 3. The most degradation due to the user is found in the voice scenario with the user head and hand, where the efficiency dropped about $70 \%$. Notice that due to the symmetric nature of the MIMO antenna design, the performance of the two antennas is comparable, especially in the data position where the user effects is almost symmetric too due to the symmetric positioning of the user's two hands.

3.1.3. Radiation Properties. The 3D radiation patterns for both antennas in the two designs were simulated. Figure 6 shows these simulations for the MIMO design in the different user positions investigated. These patterns show the general impact that the user has on the antenna far-field behaviour, which will come in handy when explaining the user effects in the channel statistics. However, a conclusive analysis cannot be presented based only on the field patterns, especially when it comes to the energy lost in the human body and correspondingly the remaining radiated power. Therefore, we use the mean effective gain (MEG) for this analysis [13],

$$
\begin{aligned}
& \text { MEG } \\
& \quad=\oint\left[\frac{\mathrm{XPR}}{1+\mathrm{XPR}} P_{\theta}(\Omega) G_{\theta}(\Omega)+\frac{1}{1+\mathrm{XPR}} P_{\phi}(\Omega) G_{\phi}(\Omega)\right] d \Omega .
\end{aligned}
$$

In (4), $G(\Omega)$ and $E(\Omega)$ are the active gain and the active electric field patterns, respectively. The angular density function is denoted by $P(\Omega)$, and XPR is the cross-polarization power ratio.

Table 4 shows the variation of MEG for studied scenarios for the MIMO design. As expected, the highest gain is in the free space scenario. The lowest gain can be seen in the voice scenario where the head and hand position as well as in the data position.

Table 5 shows the TRP and the power loss $\left(\mathrm{P}_{\mathrm{L}}\right)$ for the investigated scenarios of the MIMO design. The power loss is mostly noticed in the voice position. This is also reflected in the TRP being the lowest in this position and it explains the reduced coupling in this scenario mentioned earlier in Section 3.1.1. The TRP is now less due to the increased losses and this increases the reflected power back into the frontend. Therefore, the interaction, and hence the coupling, between the antennas is less.

The measured far field radiation patterns are given in Figure 7 for each of the MIMO antennas. The patterns are measured in $\mathrm{dBm}$ in the azimuth plane $(x-y)$ for both antennas. These show the interference of the head phantom in changing the antenna pattern in the free space scenario to become a more directed pattern in the presence of the user head. The effect of this change will be further discussed when we analyze the effects of changing the user's orientation on the MIMO channel.

3.2. Channel Statistics. We covered in Section 3.1 several aspects of the user effects on the MIMO antenna system performance by looking at the antenna parameters. We now incorporate these aspects into effects seen in the MIMO channel. Five aspects of the user effects within the channel are discussed in this section. The analyses are made based on the average channel power, the average channel capacity, the channel outage capacity, the correlation factor, and the cumulative distribution function of the channel capacity. These are investigated in the presence of the user in the three different usage scenarios shown in Figures $1(b)-1(d)$ and are compared to the free space scenario shown in Figure 1(a). We also compare with the Rayleigh fading channel which serves as a common reference to other systems.

3.2.1. Channel Average Capacity. In the absence of the channel state information at the transmitter, it is optimal to allocate power equally over all antennas. Thus, for a given total transmit power constraint of $P$; the channel capacity upper limit is given by [1]

$$
C=\log _{2}\left(\operatorname{det}\left(\mathbf{I}+\left(\frac{\mathrm{SNR}}{2}\right) \mathbf{H H}^{\dagger}\right),\right.
$$

where I is an $2 \times 2$ identity matrix, and SNR is the signal-tonoise ratio defined at the transmitter.

Examining the channel average capacity in the tested scenarios given in Figure 8, it is noticed that the lowest capacity is associated with the data position as compared to the other scenarios. The highest capacity is seen in the free space scenario and the talk scenario when only the user head is present. The head can be seen as a reflector to the antenna resulting in a more directional pattern and an improved gain [15] in some of the user orientations. This relates to the higher MEG that was measured for this scenario in Section 3.1.3.

The orientation of the device has the least effect on the capacity in the data scenario as the hands wrap around the antennas; see also Figure 6(d). While a change of up to $1 \mathrm{bit} / \mathrm{Sec} / \mathrm{Hz}$ can be seen in the voice position with respect to the device orientation. The effect of the orientation in the voice scenario can be attributed to the fact that the user's head and hand make the radiation pattern more directional in certain orientations, and therefore more dependent on orientation. This can be seen in the radiation patterns in Figure 6(c).

3.2.2. Channel 5\% Outage Capacity. Figure 9 shows the outage capacity results for the MIMO design. Similar observations made on the average capacity can be made here on the outage capacity.

\subsubsection{Average Channel Power Factor. The average channel} power factor is defined as the amount of power transferred through the channel from one end of the communication link to the other. It implicitly includes the antenna properties such as the gain as well as any losses introduced due to the presence of the user, which are incorporated in the antenna field pattern. The average channel power factor is computed as the inverse of the channel normalization factor in (2).

Figure 10 shows the channel average power factor for both designs in the different tested usage scenarios. Notice 


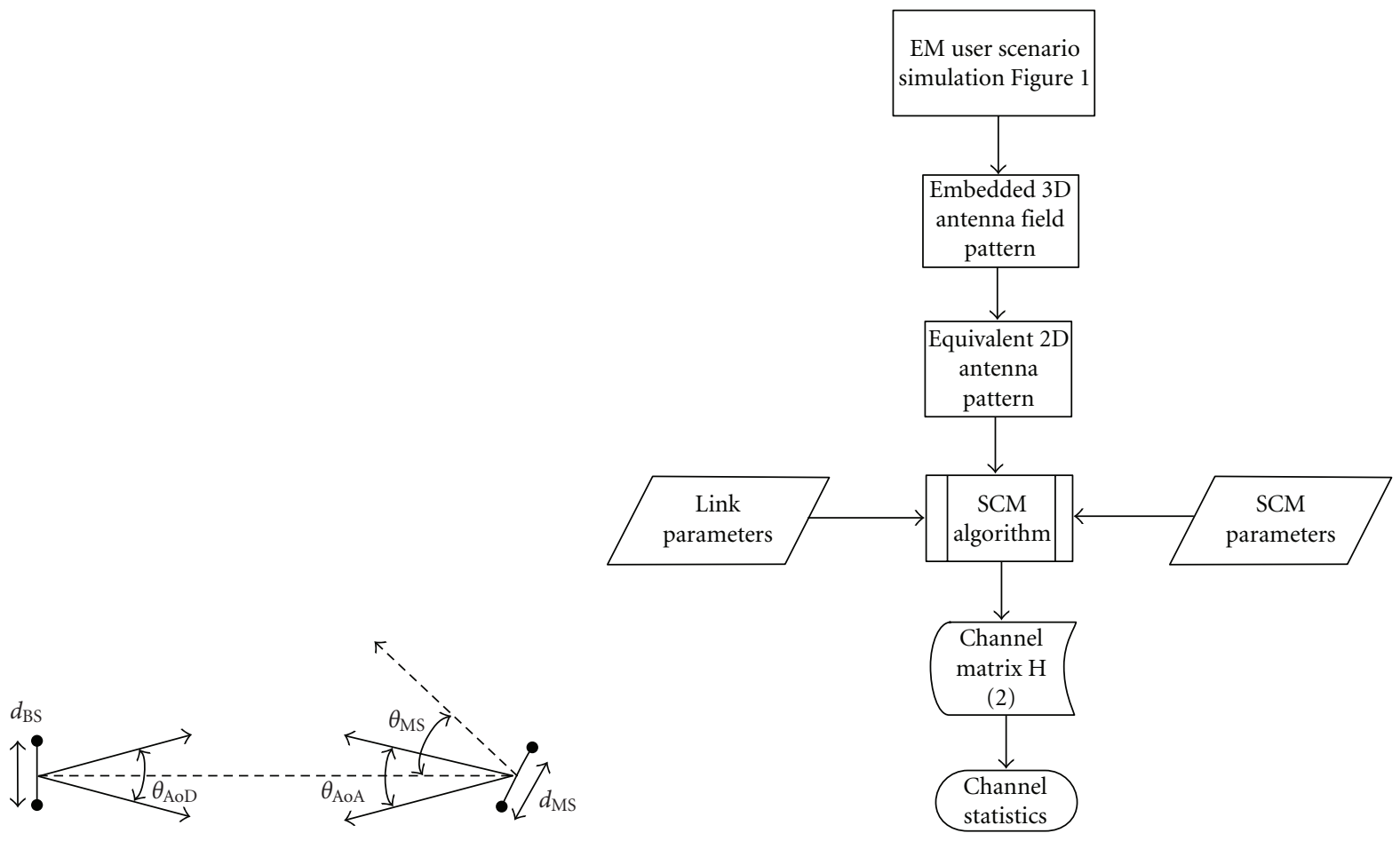

(a)

(b)

FIgURE 2: Simulation settings: (a) BS and MS array orientation, and (b) flowchart showing the SCM-EM integration.

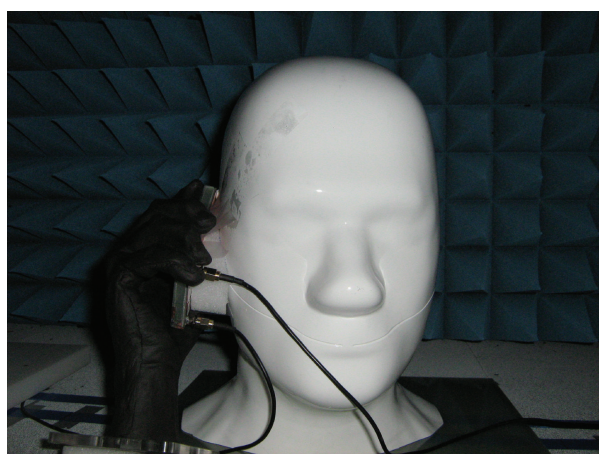

(a)

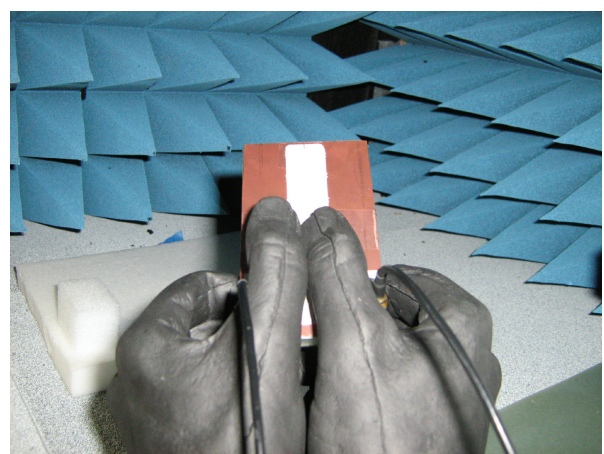

(b)

Figure 3: Lab measurements' set-up for the MIMO design, (a) voice position with the handset, head, and the hand, and (b) two hands and handset in data position.

that although the TRP as given in Section 3.1.3 for the free space scenario is larger than that in the presence of the user head; however, in specific orientations, the channel power is in fact larger with the user head than without it-see, for example, at orientations $180^{\circ}$ and $135^{\circ}$. This is a result of the propagation model and of the orientation. It demonstrates the importance of including both antenna parameters and channel simultaneously when evaluating an antenna design. This finding coincides with the results of [15] where the presence of the head in fact increased the two-antenna system capacity over the free space scenario. The reasoning behind this comes from the fact that the head acts as a reflector to the antenna pattern making it more directional in certain orientations. This increases the antenna gain, and hence the channel power, in that direction.

From an antenna perspective, the lowest TRP was found in the voice scenario as compared to the data scenario, that is, in the presence of the user head and hand. However, the propagation environment offered this scenario an advantage over the data scenario in improved channel power for some orientations. Therefore, the channel multipath has a significant impact on MIMO antenna performance. A rich multipath channel can complement a less than optimum MIMO antenna design. It can also spar it even more in a poor multipath channel. It is worth noting here that the balance between the effects of the channel and those of the antenna 


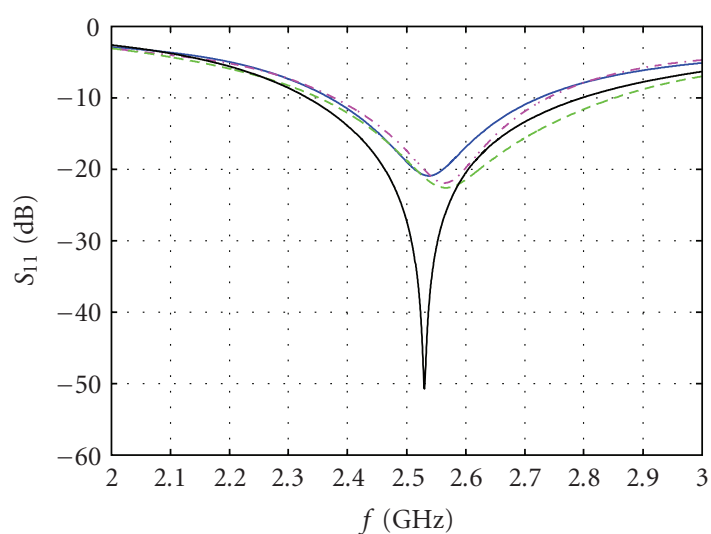

(a)

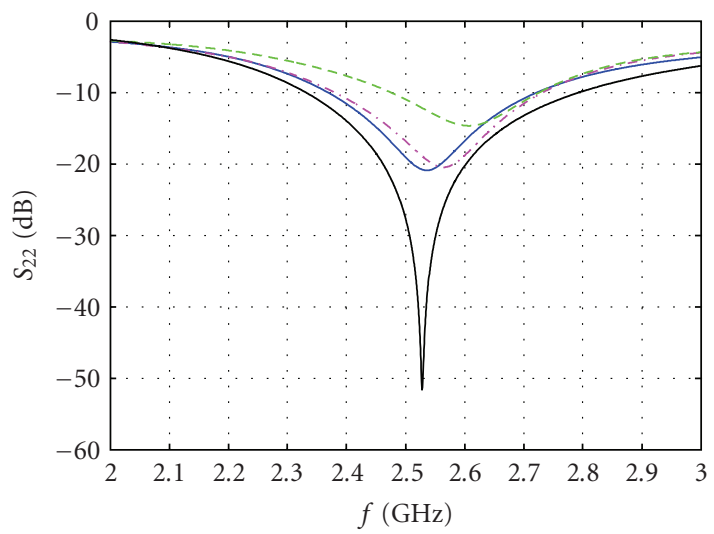

(c)

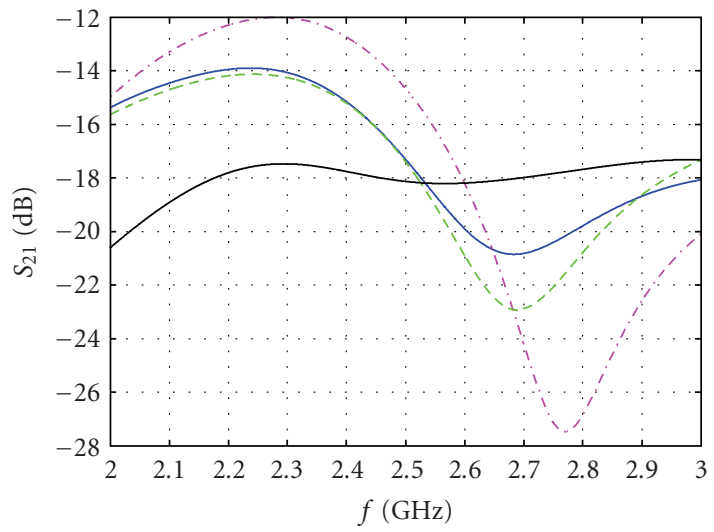

- Free space

-. - Head (tilt position)

- - - Head and hand (tilt position)

— Data position

(e)

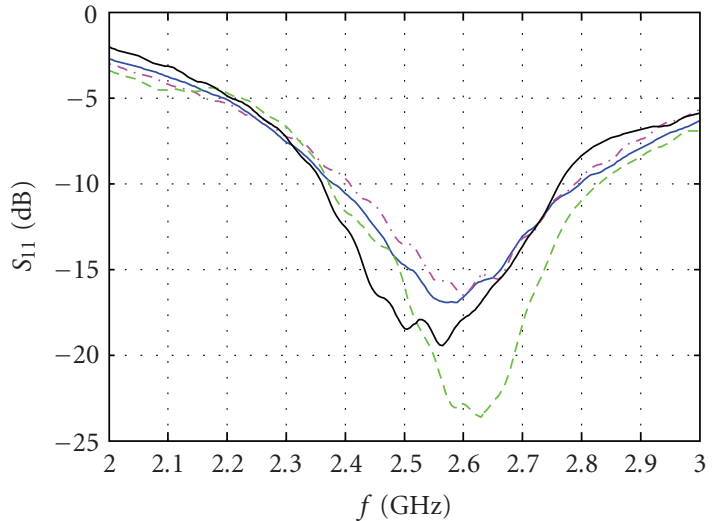

(b)

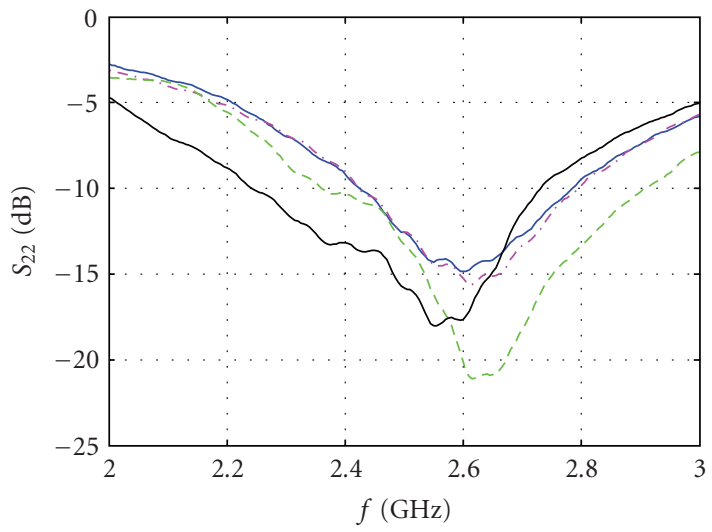

(d)

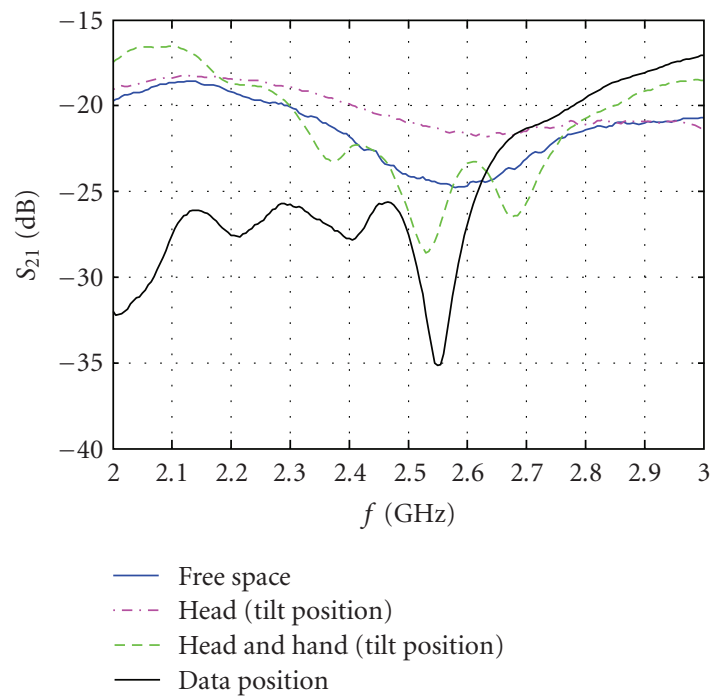

(f)

FiguRE 4: $S$-parameters for PIFA design (a) simulated $S_{11}$, (b) measured $S_{11}$, (c) simulated $S_{22}$, (d) measured $S_{22}$, (e) simulated $S_{21}$, (f) measured $S_{21}$.

in determining the overall system performance is frequency dependant [6].

In general, almost half the channel power is lost due to the presence of the user as compared to the free space case.
3.2.4. Correlation. The channel correlation results are given in Figure 11 for the signals received on the two mobile antennas. Notice that the MIMO design had a consistently higher correlation values in the data position for all the 


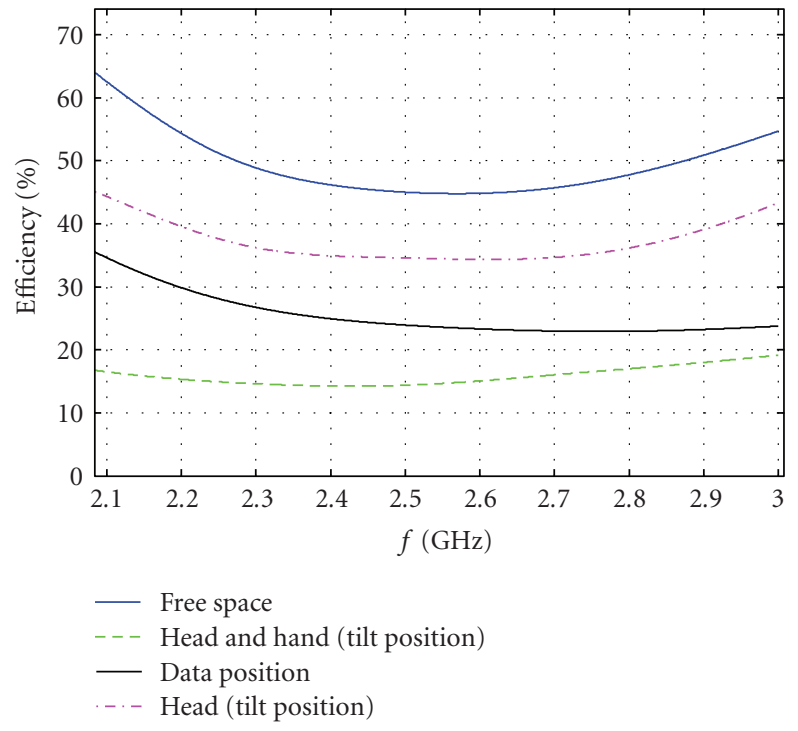

(a)

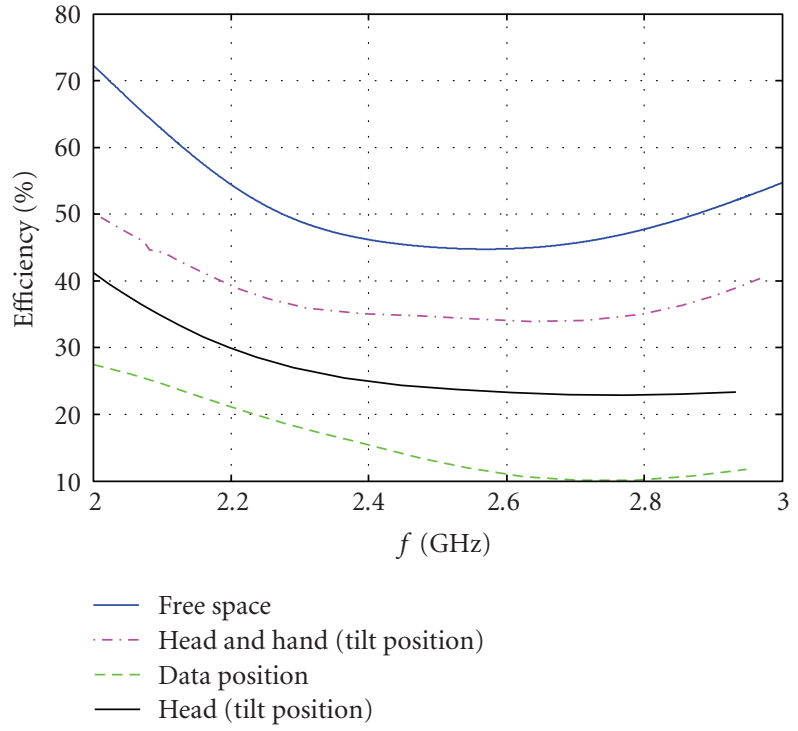

(b)

Figure 5: Efficiencies for both antennas in MIMO design, (a) antenna 1, and (b) antenna 2.

examined orientations. This can be attributed to the fact that these MIMO antennas were both oriented vertically in the device. Hence, their patterns are more likely to be correlated. In addition, the user's hands almost completely cover the MIMO antennas in the data scenario therefore reducing the pattern diversity, see Figure 6(d) for illustration.

The results also show that correlation changes meaningfully as the user changes orientation in all the tested scenarios. In some orientations, the user can in fact provide diversity in the antenna pattern and hence reduce the channel correlation to a point that is even below the correlation of the free space scenario. This can be seen looking at the radiation pattern plots of Figure 6. See for example the voice mode, Figure $6(\mathrm{c})$, the user's hand deforms the radiation pattern into two main beams introducing pattern diversity.

The head position showed the largest correlation, which is expected due to the beam-like antenna pattern that resulted from the presence of the user head, see Figure 7(b).

Notice that the correlation values in all user and free space scenarios for the MIMO design is acceptable and below 0.4 , that is, below the 0.5 correlation limit described for the mobile device in the literature.

3.2.5. Cumulative Distribution Function of the Channel Capacity. The combined effects of both the channel correlation and the channel power loss due to the presence of the user can be shown in the capacity CDF curves given in Figure 12. The results are given for the data and for the voice scenarios, they are compared to the no user scenario and to the ideal Rayleigh fading channel.

As shown, the probability of achieving a capacity value lower than a given limit increases moving from the free space scenario to the data scenario. For example, the probability of having a channel capacity lower than $10 \mathrm{bits} / \mathrm{Sec} / \mathrm{Hz}$ is almost zero in the Rayleigh channel. This probability becomes less than $10 \%$ in the free space scenario, and less than $25 \%$ in the voice position while less than $35 \%$ in the data position.

Notice that the effect of the orientation increases as the capacity increases in the free space scenario. The effect of the user orientation is more significant in the voice position than in the data position. This can be explained by looking at the radiation patterns shown in Figure 6. The user's head and hands contribute to deforming the antenna radiation pattern and distributing it in more directions. Hence, the probability that user enjoys an increase in capacity when oriented in direction(s) that take advantage of this pattern deformation is higher.

The data usage scenario, in general, is the least affected by the user's orientation. This is attributed to the fact the user hands cover, in the most part, the antennas absorbing the most power radiated such that the remaining power is not as much affected by the channel environment.

3.3. Link Perspective. We analyze at the link level and investigate the performance of the system based on antenna properties and the channel statistics presented in Sections 3.1 and 3.2, respectively, in the different usage scenarios. A MIMO-OFDM system based on 3GPP Release 8 standard [16] is used for the analysis. The channel matrices output from the integration of the EM and the SCM model, see Figure 2, are input into the link simulator. The evaluation is based on the throughput of the system in the different usage scenarios. Table 6 summarizes the simulation parameters of the link level simulator.

Figure 13 shows the block error rate (BLER) for transmission of 5 resource blocks (RBs) by using transmit diversity technique in an MIMO system with $M=2$ transmit and $N=2$ receive antennas $(M \times N$ system $)$. The effect of different usage scenario at 0 degree user orientation is simulated. It can be seen that due to the presence of a user 


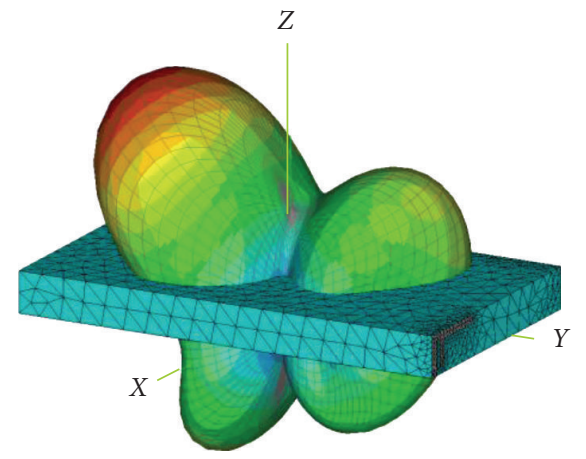

(a)

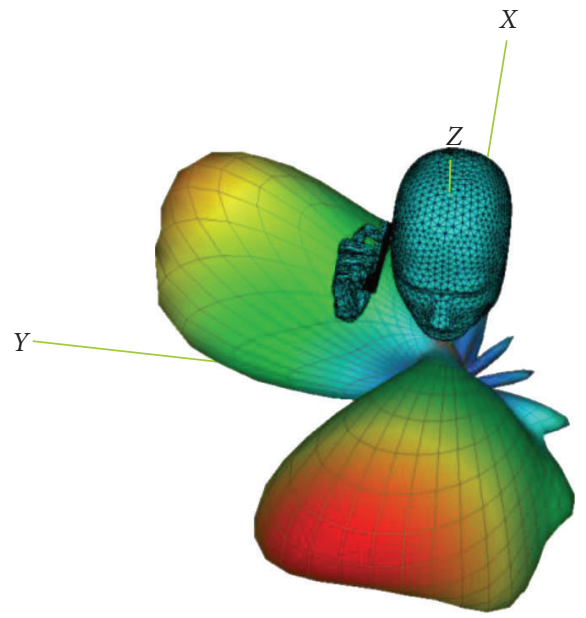

(c)

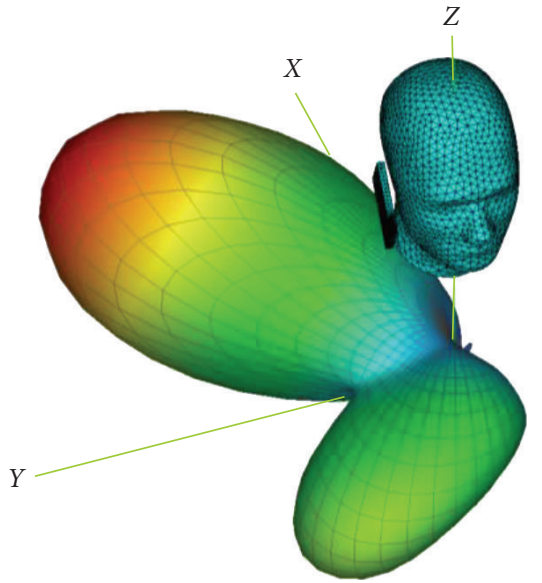

(b)

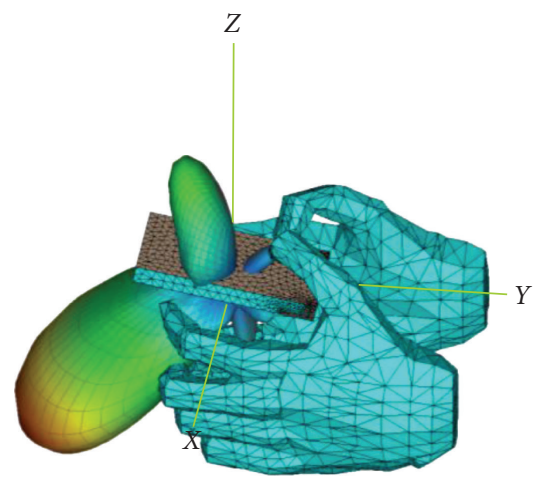

(d)

FIgure 6: Total 3D radiation patterns of the MIMO design: (a) without the user, (b) voice position with the user head, (c) voice position with the user head and hand, and (d) data position with the user hands.

the performance of our system can be dramatically changed in the order of $5 \mathrm{dBs}$ in performance difference moving from the best performance without the user to the weakest performance seen in the data position at BLER of $10^{-2}$. The throughput performance of this system is depicted in Figure 14, which aligns well with the correlation results of Figure 13. The best performance is for the case without user. Notice that it takes $5 \mathrm{dBs}$ more to achieve $1.25 \mathrm{Mbps}$ moving from an FS scenario to the head only scenario.

The simulations are repeated for the same settings but with four antennas on the BS, that is, for a $4 \times 2$ system, with transmit diversity also given in Figure 14. The results show that having more antennas helps reduce the performance variation in the different usage scenarios compared to the $2 \times 2$ case. Additionally, the link throughput showed a better performance of the voice scenario over the data scenario in a $2 \times 2$ system, where this situation is flipped in the $4 \times 2$ case.

Figure 15 introduces the link throughput performance of a $4 \times 2$ MIMO system with spatial multiplexing. As seen, at 2 Mbps throughput, we need $4 \mathrm{~dB}$ more in $\mathrm{H}$ scenario to get the same performance without the user being present. Notice that an increase of $6 \mathrm{~dB}$ and $10 \mathrm{~dB}$ is needed in the voice and in the data scenarios, respectively, to obtain the same performance in the absence of the user.

We examine the user effects in the different LTE transmission schemes through a comparison between spatial multiplexing and transmit diversity in a $4 \times 2$ MIMO system in different usage scenarios in Figure 16. Notice that in the absence of the user, throughput improves at the high SNRs for spatial multiplexing while at low SNRs transmit diversity shows a better performance. Now, if we consider different usage scenarios, we observe that it is possible that we do not need to change our transmission scheme at reasonable ranges of SNR. Figure 16 suggests that transmit diversity is performing better than spatial multiplexing in the desired range of SNR while spatial multiplexing is performing better than transmit diversity in another usage scenario. It is worth mentioning here that not only the transition point has been changed, but also the behaviour of the two curves compared to each other has been changed. This can be 

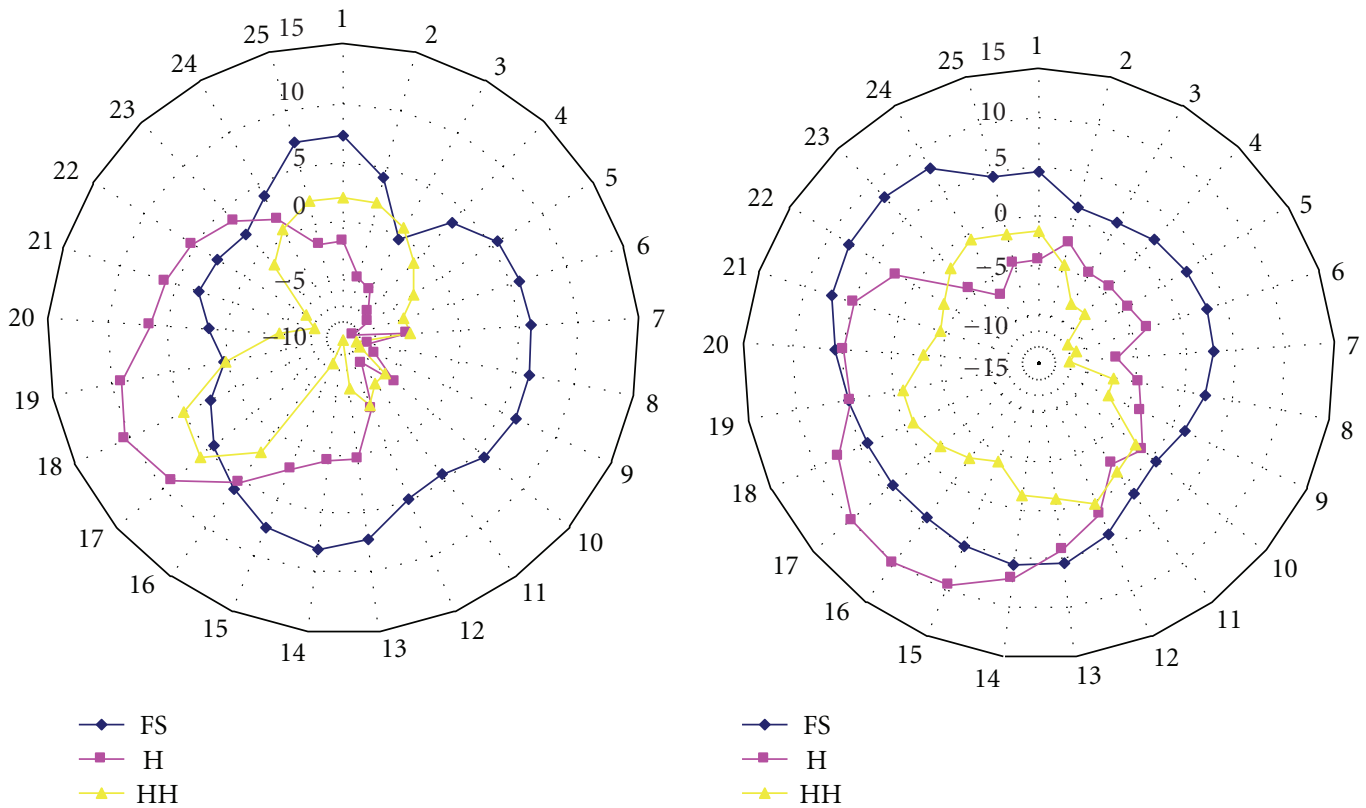

(a)

(b)

FIgURE 7: Measured radiation patterns (in $\mathrm{dBm}$ ) on the azimuth plane $(x-y)$ for (a) antenna 1 and (b) antenna 2-FS: free space, H: voice scenario with only head, HH: voice scenario with head and hand.

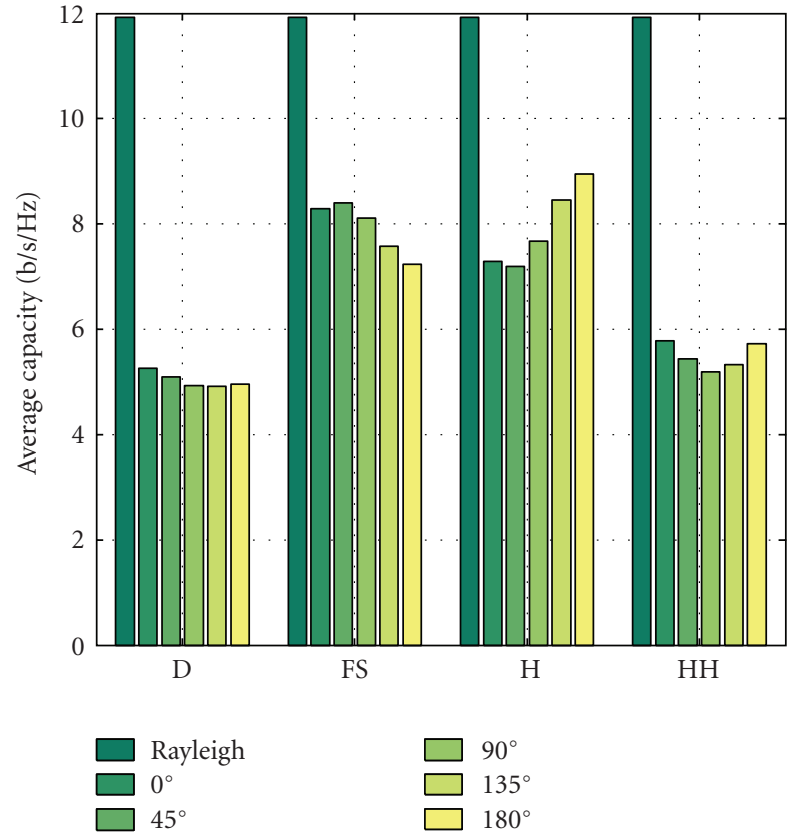

FIGURE 8: Average channel capacity in the different usage scenarios at different user orientations.

easily explained by the radiation pattern in Figure 6(d). As it can be seen, the presence of the user's hands changed the radiation pattern of the wireless device (compared to Figure 6(a)), such that there is almost one dominant lobe for the pattern. In Figure 6(a), there are two dominant lobes. However, in the data position, we almost lose one lobe, which does not affect the transmit diversity performance; as in transmit diversity concept we provide multiple choices by

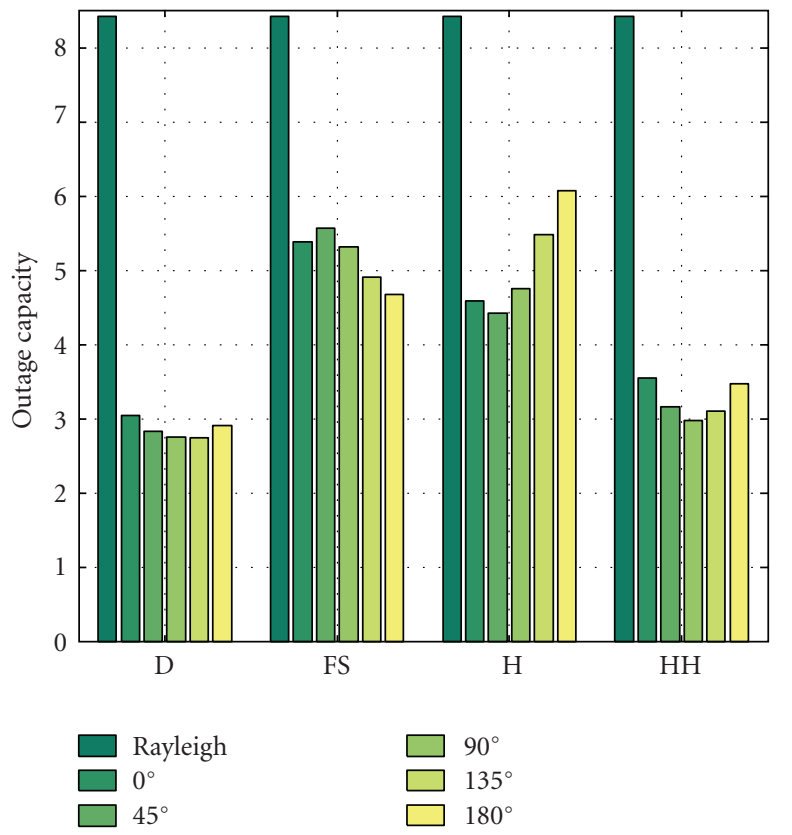

FIGURE 9: Outage channel capacity in the different usage scenarios at different user orientations.

transmitting multiple similar data signals, so at least one of them can be received. Spatial multiplexing scheme relies on both lobes for a good performance.

\section{Conclusions}

We thoroughly investigate in this paper the effects of the user's presence on the performance of an MIMO device 


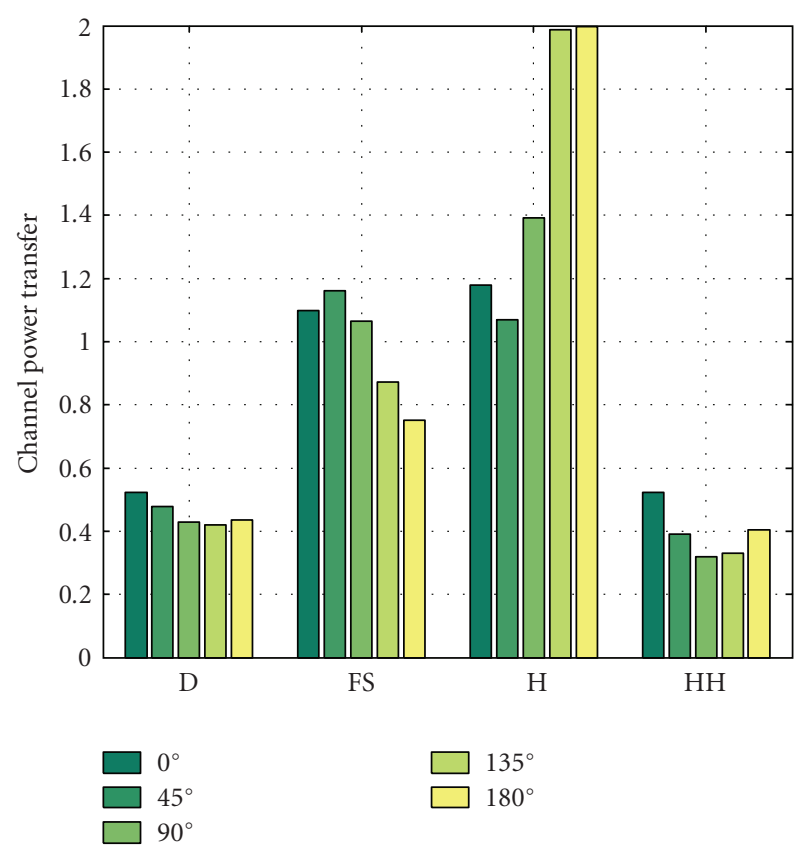

Figure 10: Average channel power factor in the different usage scenarios at different user orientations.

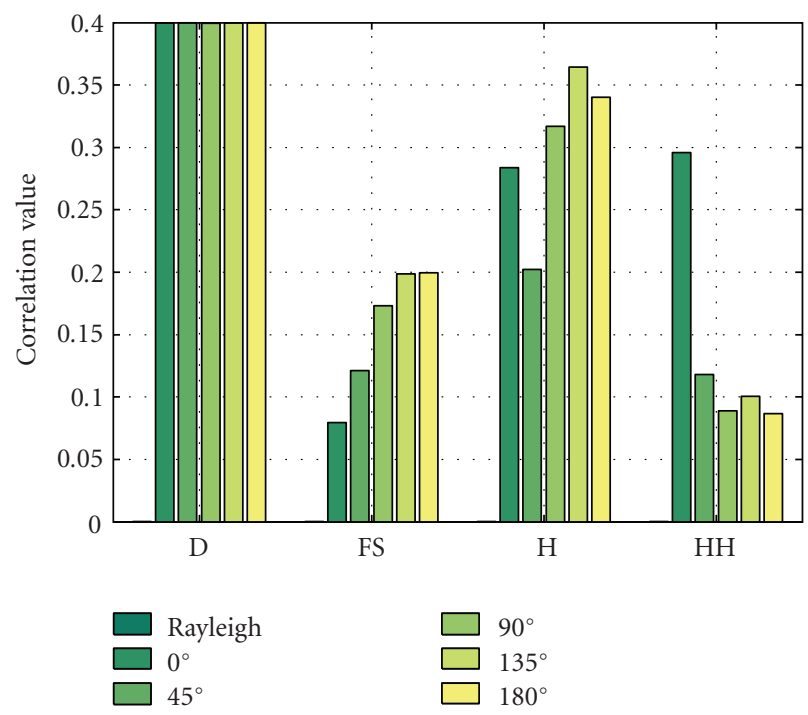

FIgURE 11: Channel correlation values in the different usage scenarios at different user orientations.

starting from an antenna perspective and moving to the link perspective. The investigation starts by looking at the user effects on the MIMO antenna performance as a deterministic element. We then extend the investigation to integrate the antenna characteristics into the statistical propagation channel environment. The MIMO channel is built by integrating the deterministic $3 \mathrm{D}$ properties of the antennas' and their near-field user effects into the 3GPP spatial channel model (SCM) for a macrocell environment. Two usage scenarios are examined, voice and data positions. The performance of these scenarios is compared to that in

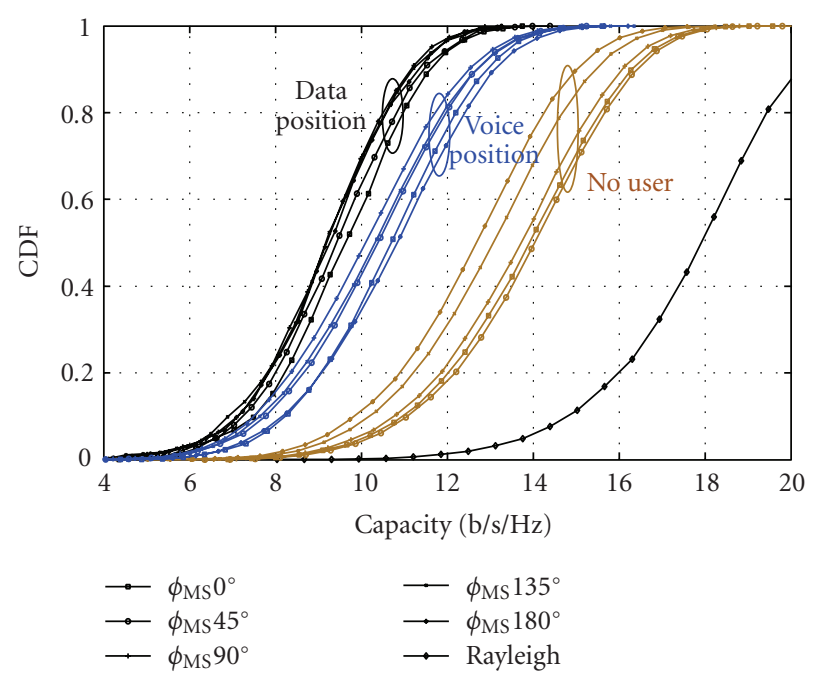

Figure 12: Capacity CDF at SNR $30 \mathrm{~dB}$ in the different usage scenarios at different orientations of the user.

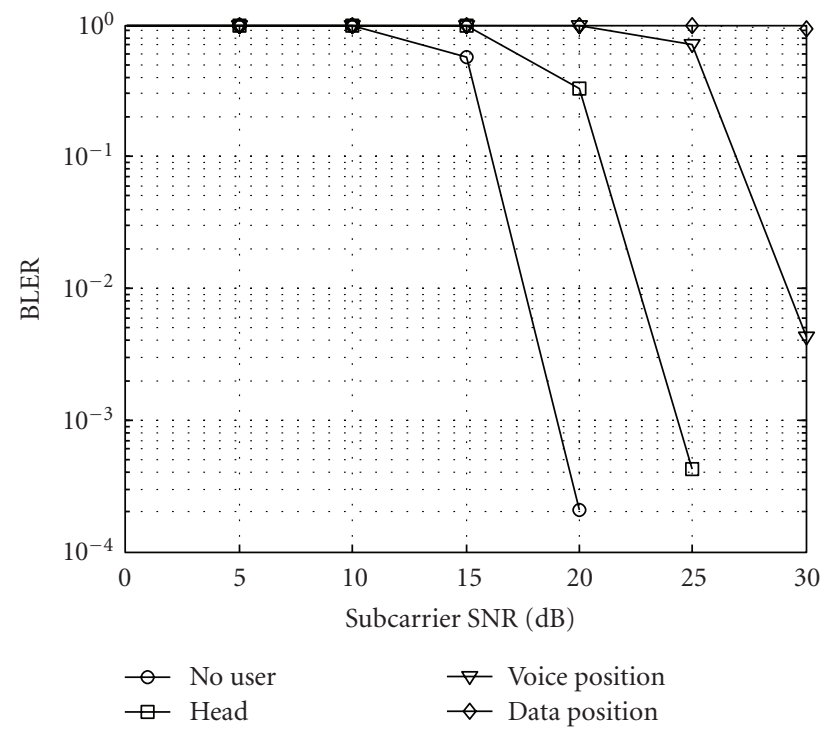

FIGURE 13: BLER in different usage scenarios for a $2 \times 2$ MIMO system with transmit diversity.

the absence of the user. Our investigations showed that the user caused a power loss that translates into a reduction of up to $70 \%$ in the antenna efficiency and up to $4 \mathrm{~dB}$ loss in the gain. The power loss also had a negative effect on the total radiation power. This reduction in the power translated into a reduction in the channel capacity and in the link throughout. It was found that the coupling between the two MIMO antennas reduced as a result of this reduction in the radiated power. This reduction in the antenna mutual coupling translated into increased channel correlation, which mostly appeared in the data position. Although the most degradation in the antenna performance was noticed in the voice position having the user head and hand present, yet the most degradation in the MIMO channel statistics was seen in the data position. This is attributed to the pattern diversity 


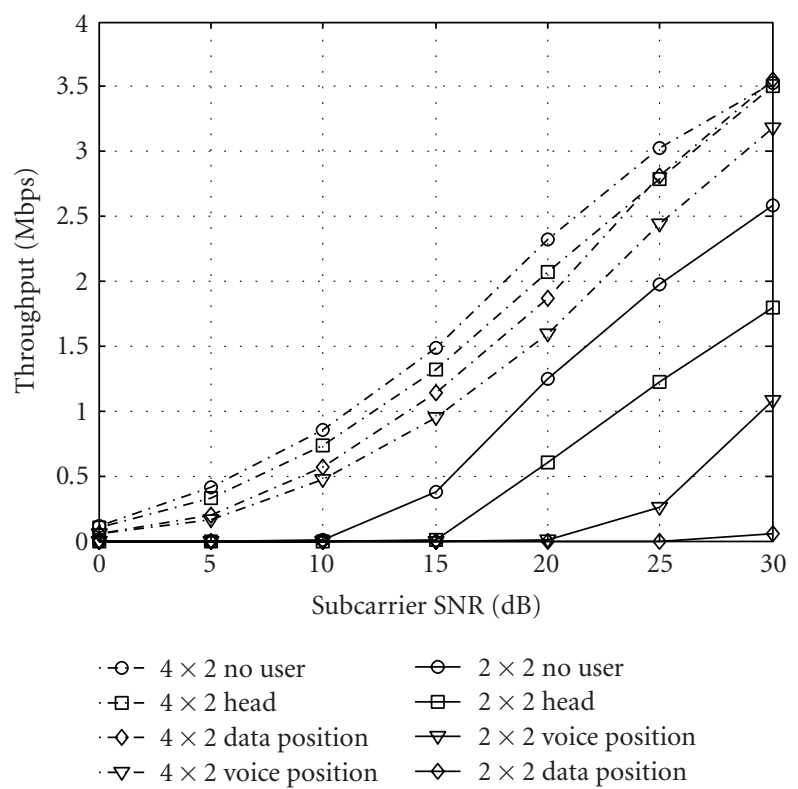

FIGURE 14: Link throughput in different usage scenarios for an $\mathrm{M} \times$ N MIMO system with transmit diversity.

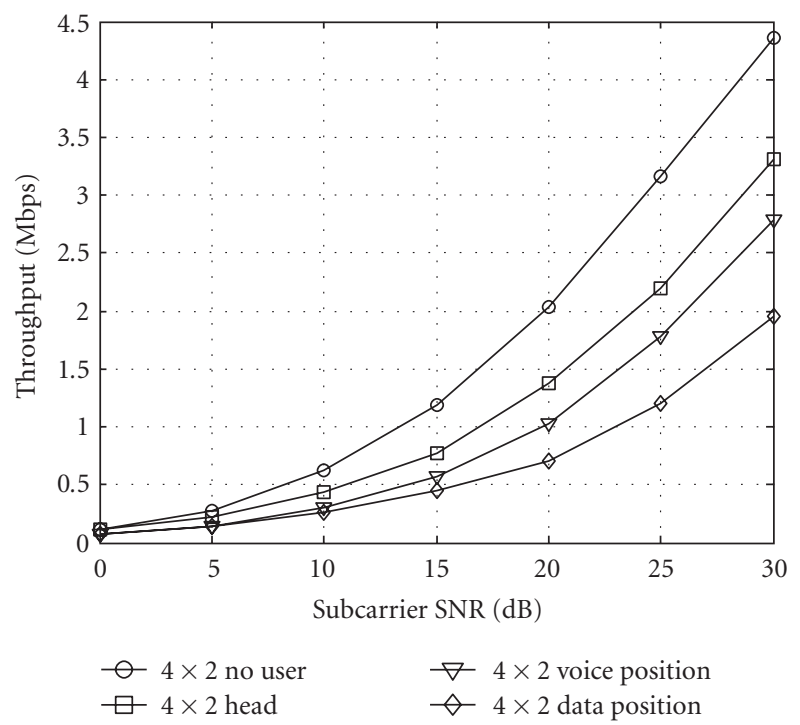

FIGURE 15: Link throughput in different usage scenarios for a $4 \times 2$ MIMO system with spatial multiplexing.

that is achieved in the voice position, which compensated for the loss in the power.

Finally, we extend the investigation to relate the user effects and the antenna performance to the link performance. We show that there are a reasonable ranges of SNR in which the difference in performance is minor when changing between the different LTE transmission schemes in the presence of the user. However, the performance could change meaningfully with the different transmission schemes outside of these ranges. To this effect it was found that the transmit diversity scheme performs better than spatial multiplexing in a given SNR range and usage scenario

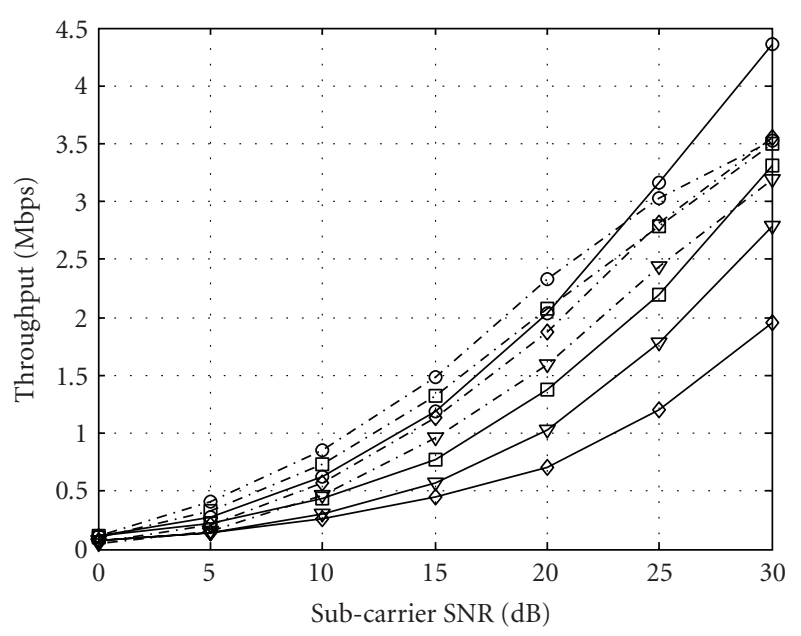

$$
\begin{array}{ll}
Ð 4 \times 2 \text { no user } & \wp-4 \times 2 \text { no user } \\
\square-4 \times 2 \text { head } & Ð-4 \times 2 \text { head } \\
\nabla 4 \times 2 \text { voice position } & \diamond-4 \times 2 \text { data position } \\
\vartheta 4 \times 2 \text { data position } & \nabla^{-} 4 \times 2 \text { voice position }
\end{array}
$$

Figure 16: Comparison of spatial multiplexing and transmit diversity performance in (a) FS, (b) $\mathrm{H}$, (c) $\mathrm{HH}$, and (d) D for a $4 \times 2$ MIMO system.

while spatial multiplexing scheme outperforms the transmit diversity scheme in another usage scenario.

\section{Acknowledgments}

The authors would like to thank Reza K. Amineh for his help with some of the EM simulations and would like to thank Eoin Buckley for his comments and support.

\section{References}

[1] G. J. Foschini and M. J. Gans, "On Limits of wireless communications in a fading environment when using multiple antennas," Wireless Personal Communications, vol. 40, no. 6, pp. 311-335, 1998.

[2] H. R. Chuang, "Human operator coupling effects on radiation characteristics of a portable communication dipole antenna," IEEE Transactions on Antennas and Propagation, vol. 42, no. 4, pp. 556-560, 1994.

[3] K. Tsunekawa and A. Ando, "Advanced wire grid method for solving the scattered field of a lossy dielectric object," IEEE APS International Symposium Digital, vol. 2, pp. 797-800, 1992.

[4] S.-I. Watanabe, "Characteristics of the SAR distributions in a head exposed to electromagnetic fields radiated by a handheld portable radio," IEEE Transactions on Microwave Theory and Techniques, vol. 44, no. 10, pp. 1874-1883, 1996.

[5] C. Li, E. Ofli, N. Chavannes, and N. Kuster, "The influence of the user hand on mobile phone antenna performance in data mode," in Proceedings of the 3rd European Conference on Antennas and Propagation, (EuCAP '09), pp. 449-452, Berlin, Germany, March 2009.

[6] S. M. Ali, "Fundamentals for a pragmatic MIMO performance evaluation," in MIMO Systems, Theory \& Applications, pp. 395-412, INTECH, 2011. 
[7] K. Ogawa, T. Matsuyoshi, and K. Monma, "An analysis of the performance of a handset diversity antenna influenced by head, hand, and shoulder effects at $900 \mathrm{MHz}$. II. Correlation characteristics," IEEE Transactions on Vehicular Technology, vol. 50, no. 3, pp. 845-853, 2001.

[8] Technical Specifications Group Radio Access Network, "Spatial channel model for multiple input multiple output (MIMO) simulations-TR25.996," 3GPP Partners, 2006.

[9] IEEE recommended practice for determining the peak spatialaverage specific absorption rate (SAR) in the human head from wireless communications devices: measurement techniques, IEEE Standards $1528^{T M}, 2003$.

[10] "Body tissue dielectric parameters," http://www.fcc.gov/oet/ rfsafety/dielectric.html.

[11] P. Suvikunnas, J. Salo, and P. Vainikainen, "Impact of power normalization in experimental MIMO antenna performance studies," IEEE Antennas and Wireless Propagation Letters, vol. 6, pp. 43-46, 2007.

[12] $\mathrm{FEKO}^{\circledR}$, User Manual, Suite 5.3, EM Software \& Systems-S.A. (Pty) Ltd, 32 Techno Lane, Technopark, Stellenbosch, 7600, South Africa, August 2006.

[13] T. Taga, "Analysis for mean effective gain of mobile antennas in land mobile radio environments," IEEE Transactions on Vehicular Technology, vol. 39, no. 2, pp. 117-131, 1990.

[14] H. Kanj, P. Lusina, S. M. Ali, and F. Kohandani, "A 3Dto-2D transform algorithm for incorporating 3D antenna radiation patterns in $2 \mathrm{D}$ channel simulators," IEEE Antenna and Propagation Wireless Letters, pp. 815-818, 2009.

[15] S. M. Ali, S. Qu, and F. Kohandani, "Capacity of an antenna system in the transmission mode with near-field effects," in Proceedings of the Annual Review of Progress in Applied Computational Electromagnetics (ACES '08), Niagara Falls, Canada, April 2008.

[16] TSG-RAN TR 36.211, "Evolved universal terrestrial radio access (E-UTRA); physical channels and modulation," Version 8.8.0, September 2009. 

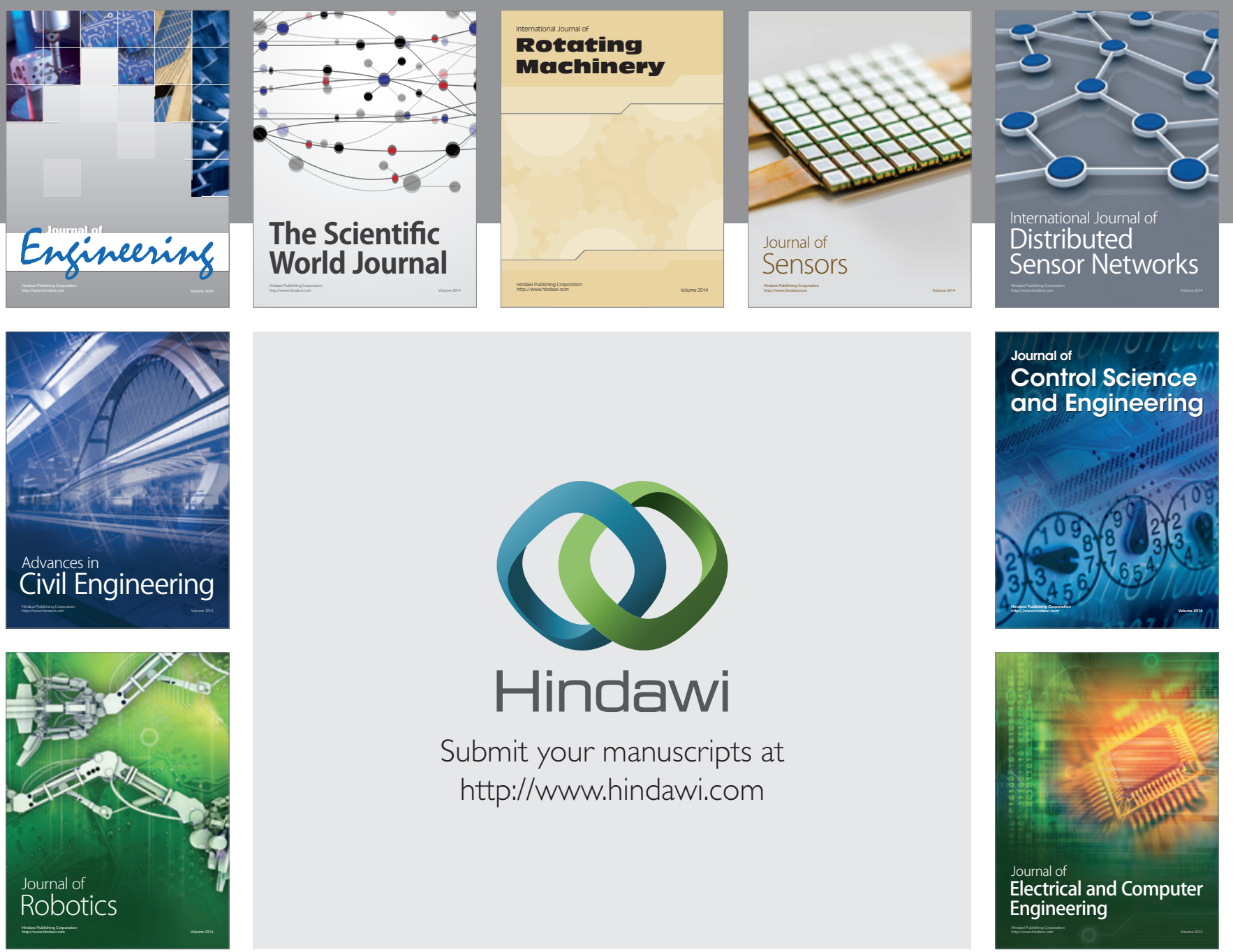

Submit your manuscripts at

http://www.hindawi.com
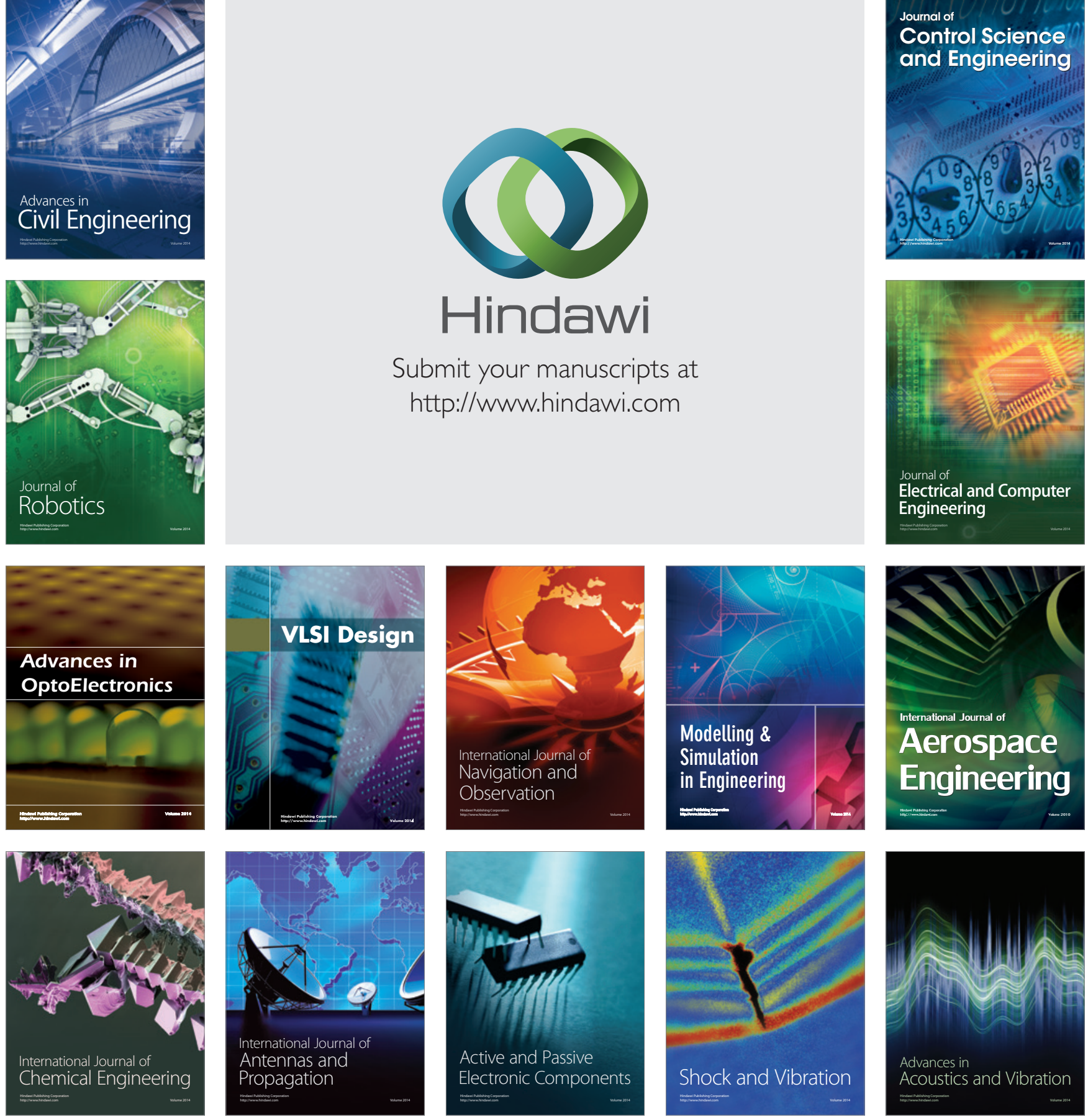